\title{
Alcohol-Associated Immunoglobulin A Vasculitis: A Case Report and Review of the Literature
}

\author{
Pallavi Basu ${ }^{a} \quad$ Eleanor Russell-Goldman ${ }^{b}$ Rosalynn M. Nazarian ${ }^{c}$ \\ Shinjita Das ${ }^{d}$ \\ a School of Medicine, University of California San Diego, La Jolla, CA, USA; ${ }^{b}$ Department of \\ Pathology, Brigham and Women's Hospital, Boston, MA, USA; 'Department of Pathology, \\ Massachusetts General Hospital, Boston, MA, USA; ${ }^{\mathrm{d}}$ Department of Dermatology, \\ Massachusetts General Hospital, Boston, MA, USA
}

\section{Keywords}

Alcohol · Idiopathic $\cdot$ Leukocytoclastic vasculitis

\section{Abstract}

Immunoglobulin A (IgA)-mediated leukocytoclastic vasculitis is a cutaneous small-vessel vasculitis characterized by skin findings of palpable purpura. It may occur secondary to infections, neoplasms, drugs, and systemic conditions, although it is most commonly idiopathic. A known, but rare, trigger for IgA vasculitis is alcohol consumption. We present a case of a man with IgA vasculitis associated with alcohol use and review the literature on alcohol-associated vasculitis. Although rarely reported, alcohol-associated IgA vasculitis is an important entity to consider for appropriate diagnosis and management of such patients.

(C) 2020 The Author(s)

Published by S. Karger AG, Basel

\section{Introduction}

Immunoglobulin A (IgA)-mediated leukocytoclastic vasculitis is a small-vessel leukocytoclastic vasculitis that can affect multiple organ systems, including the skin, gastrointestinal tract, and kidneys [1]. Formerly known as Henoch-Schoenlein purpura (HSP), IgA vasculitis occurs most commonly in the pediatric population with rare incidence in adults. The proposed etiology of IgA vasculitis is broad, and the mechanism of pathogenesis is not fully understood. It is often idiopathic but can be triggered by infection, drugs, and systemic disease [2]. A 
Fig. 1. Palpable purpura in a patient with leukocytoclastic vasculitis. Distant (a) and closer (b) views of a 43-year-old man with palpable purpura (red arrows) of the lower extremities associated with alcohol consumption. Hyperpigmented patches characteristic of stasis dermatitis are also visible on the anterior shin. A 4-mm punch biopsy was performed at site of the blue arrow.
Basu et al.: Alcohol-Associated Vasculitis
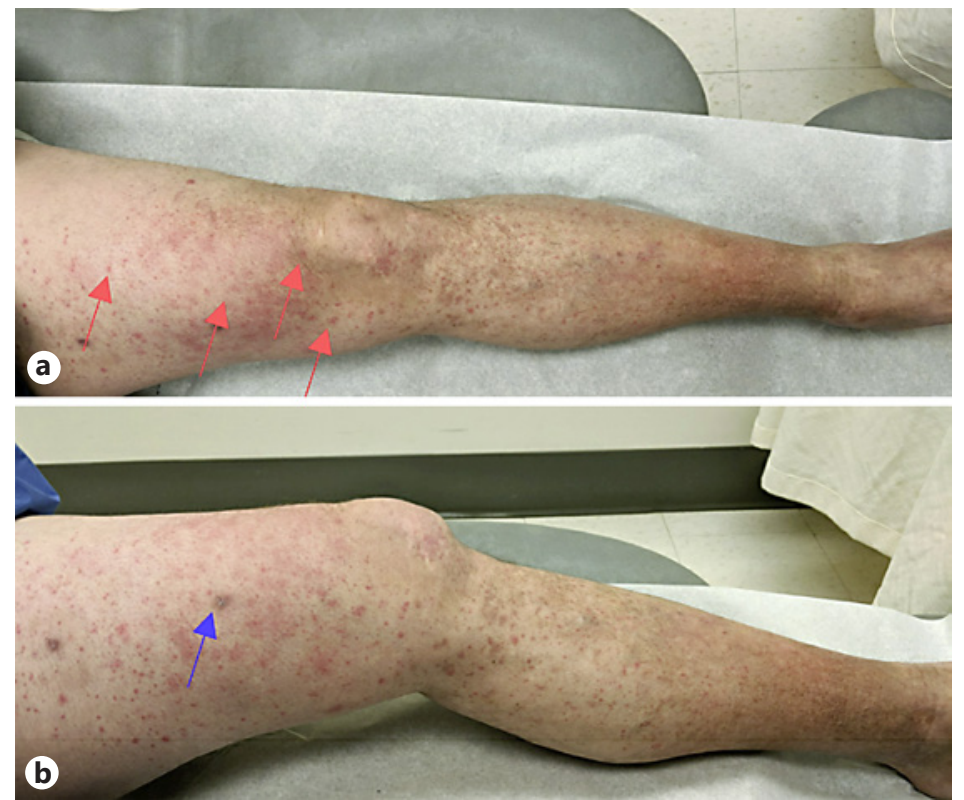

known, but rare, trigger for IgA vasculitis is alcohol consumption [3-5]. We describe the case of a 43-year-old man with recurrent IgA vasculitis following alcohol consumption and review the literature of alcohol-associated IgA vasculitis.

\section{Case Report}

A 43-year-old man presented to the clinic with episodic lower extremity rash of several years' duration. Past medical history included diverticulitis and stasis dermatitis, and he was not taking any medications. He reported that the asymptomatic rash appeared on his thighs and legs every few months, hours to days following consumption of alcoholic beverages containing hops. He did not develop this rash when he drank non-hops alcoholic beverages. The rash spontaneously resolved within days, leaving residual dark spots. Associated symptoms included swelling of his ankles, knees, and scrotum. He denied any other systemic symptoms or hematuria. There were no other known triggering factors, including no recent viral-type illness or new medications or supplements.

On cutaneous examination, he had scattered 2-3-mm pink non-blanching macules and papules extending from his bilateral lower legs to the groin (Fig. 1). He had background hyperpigmented patches on the shins, characteristic of stasis dermatitis. On further questioning, the patient described more widespread involvement on the lower extremities and previously raised quality to flat lesions.

Given clinical suspicion for leukocytoclastic vasculitis, we performed a skin biopsy. Hematoxylin and eosin staining of the specimen revealed a superficial and deep perivascular neutrophilic infiltrate with leukocytoclasia, erythrocyte extravasation, and conspicuous eosinophils (Fig. 2). Although no significant fibrinoid necrosis of the vessel walls was identified, in conjunction with the direct immunofluorescence results that revealed vessel wall immunoreactivity for IgA, the findings were consistent with an IgA vasculitis.

Laboratory studies, including a urinalysis, anti-streptolysin 0 , viral hepatitis titers, complement levels, autoantibody screen, and rheumatoid factor levels, were all negative. 

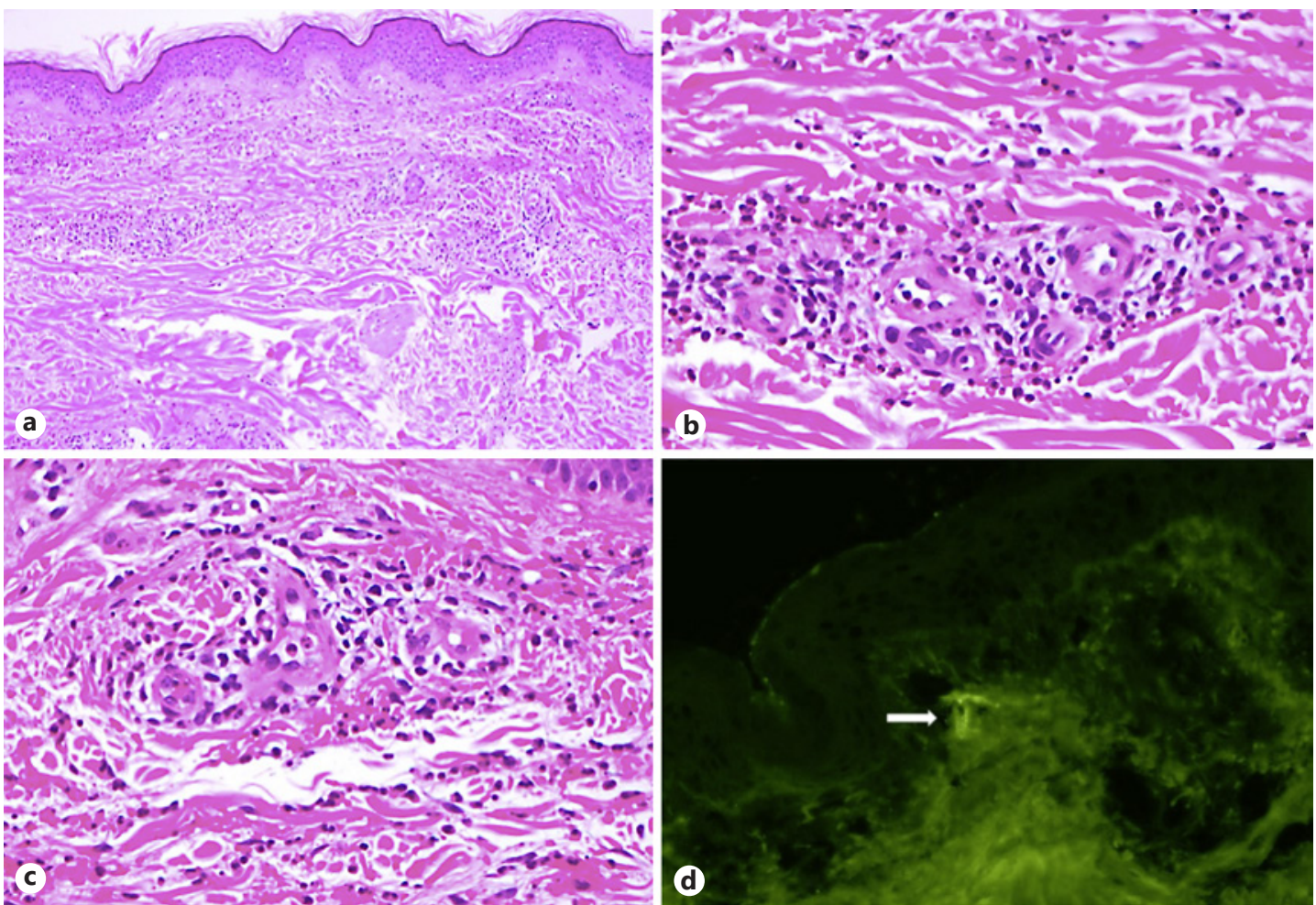

Fig. 2. Histologic and direct immunofluorescence features of IgA vasculitis. Low-power magnification (a) shows a superficial to deep dermal perivascular inflammatory infiltrate which is predominantly neutrophilic. H\&E, $10 \times$ magnification. Higher-power magnification $(\mathbf{b}, \mathbf{c})$ shows perivascular neutrophilic inflammation and leukocytoclasis with admixed eosinophils and lymphocytes as well as numerous vessels affected by neutrophilic vasculitis, with resulting red blood cell extravasation. H\&E, 40× magnification. Direct immunofluorescence (d) demonstrates granular deposition of IgA in the superficial dermal vessel walls (arrow). 40× magnification.

Triamcinolone cream provided little benefit, and the eruption was self-limited. At the 3-month follow-up, the patient reported several recurrences with alcohol use as well as a new-onset itch associated with the lesions. Because he presented to the clinic earlier on in the subsequent flare (with scrotal swelling and arthralgias), a 40-mg prednisone taper over 3 weeks was initiated with the plan to follow-up to the clinic as needed. No recurrences or complications were reported 6 months after his initial visit.

\section{Discussion/Conclusion}

IgA vasculitis, formerly known as HSP, refers to leukocytoclastic vasculitis mediated by the deposition of IgA immune complexes in vessels. Over $90 \%$ of cases occur in the pediatric population, and adult IgA vasculitis is rare. The annual incidence of IgA vasculitis is estimated at $0.8-1.8 / 100,000$ in adults and 3-26.7/100,000 in children [6]. Among adults, idiopathic cutaneous small-vessel vasculitis and IgA vasculitis are the most common subtypes of leukocytoclastic vasculitis [7]. IgA vasculitis has been diagnosed in adults up to the age of 86 years [2]. Most studies have found a slight preponderance of males to females, both in children and adults $[2,6]$. 
Basu et al.: Alcohol-Associated Vasculitis

Table 1. Characteristics of the patients with alcohol-associated IgA vasculitis

\begin{tabular}{|c|c|c|c|c|c|c|c|c|}
\hline Case & Sex & $\begin{array}{l}\text { Age, } \\
\text { years }\end{array}$ & $\begin{array}{l}\text { Type of alcohol } \\
\text { trigger }\end{array}$ & Location & $\begin{array}{l}\text { Symptomatic } \\
\text { purpura }\end{array}$ & $\begin{array}{l}\text { Extracutaneous } \\
\text { involvement }\end{array}$ & Treatment & Ref. \\
\hline 1 & $\mathrm{~F}$ & 26 & Beer, wine, liquor & Bilateral feet, legs & + & - & Avoidance only & {$[3]$} \\
\hline 2 & M & 27 & Beer & Ankles & - & - & Avoidance only & [4] \\
\hline 3 & M & 60 & Wine, vinegar & $\begin{array}{l}\text { Back, bilateral arms, } \\
\text { buttocks }\end{array}$ & - & - & Avoidance only & [5] \\
\hline 4 & M & 43 & Hops & $\begin{array}{l}\text { Bilateral lower } \\
\text { extremities }\end{array}$ & - & + & $\begin{array}{l}\text { Avoidance, TAC } 0.1 \% \\
\text { ointment, prednisone }\end{array}$ & $\mathrm{CR}$ \\
\hline
\end{tabular}

CR, current report; +, present; -, absent; TAC, triamcinolone acetonide.

While multiple pathogenic mechanisms have been implicated in cutaneous vasculitis, the cause of IgA vasculitis remains unclear, though environmental and genetic risk determinants have been suggested $[8,9]$. IgA vasculitis is most often idiopathic, though many triggers have been reported, including infections, neoplasms, inflammatory disorders, and certain drugs. In children, seasonal peaks, frequent occurrence following upper respiratory tract infection, and onset in index cases and in other family members all suggest a transmissible infectious process $[6,7]$. Possible infectious triggers in adults include, but are not limited to, Streptococcus, Mycobacterium, hepatitis B and C, Staphylococcus aureus, Chlamydia, Neisseria, and HIV [10]. In adults, alcohol-associated IgA vasculitis is a known but rare trigger; the mechanism by which alcohol may induce purpura is unclear [3, 5]. Three other biopsy-confirmed cases of alcohol-associated vasculitis have been reported in the literature and are summarized in Table 1 [3-5]. A unique aspect of our case is that our patient's alcohol-associated vasculitis seems to flare with a specific type of alcohol; namely, beers brewed with hops, flowers of the hop plant Humulus lupulus that add bitterness and floral or fruity flavors to beer.

Clinically, patients with IgA vasculitis present with palpable purpura located on the bilateral lower extremities. Symptoms may include tenderness, pain, and pruritus [7]. Two patients with alcohol-associated IgA vasculitis, including our patient, experienced symptomatic purpuric eruptions: tenderness and burning pain, respectively. The joints, gastrointestinal tract, kidneys, and occasionally other organs, can also be involved in IgA vasculitis [11]. Unlike the other cases of alcohol-associated IgA vasculitis, our patient was the only one to have extra-cutaneous manifestations; namely, scrotal edema and arthritis.

No definitive clinical criteria have been established to diagnose IgA vasculitis or HSP. The European League Against Rheumatism (EULAR) consensus criteria include palpable purpura with at least one of the following: diffuse abdominal pain, any biopsy showing IgA deposition, arthritis or arthralgias, or renal involvement (proteinuria or hematuria) [12]. However, vascular IgA deposition is non-specific for HSP diagnosis, and the utility of immunofluorescence studies for vasculitis is influenced by the clinical presentation and level of suspicion for HSP [13]. A skin biopsy within 24-48 h of the appearance of a new lesion is preferable [14]. Characteristic findings, as observed in our patient, include neutrophilic infiltration of the dermal small blood vessel walls associated with fibrinoid necrosis and disruption of the vessel wall, nuclear debris (leukocytoclasis), endothelial swelling, and extravasation of erythrocytes $[7,14]$.

Notably, many of the clinical features of IgA vasculitis and histopathologic findings of leukocytoclasis can be seen in other small-vessel vasculitides in adults. Evaluation for systemic lupus erythematosus or complement-mediated vasculitis, microscopic polyangiitis, urticarial 
vasculitis, and ANCA-associated small vessel vasculitis may be warranted $[8,9,14]$. In the presence of arthralgias, type II cryoglobulinemia may also be considered in the clinical differential diagnosis. All previously reported cases of alcohol-related IgA vasculitis, with the exception of the 26-year-old woman, underwent comprehensive laboratory evaluation that was negative for underlying disease [3]. Additionally, other alcohol-associated dermatoses, including Schamberg's disease (progressive pigmented purpura) and idiopathic thrombocytopenic purpura, may be worth consideration and further evaluation as warranted.

IgA vasculitis is often a self-limited disease, but a subset of patients experience a relapsingremitting disease course. Renal involvement, which has greater incidence in adults, is the major long-term detrimental prognostic factor [1]. None of the previously reported cases of alcohol-associated vasculitis had evidence of renal involvement. Treatment of skin disease is primarily supportive. Antihistamines are often helpful for pruritus [9, 15]. Mid-potency topical corticosteroids, as prescribed to our patient, may also have some benefit, particularly for cutaneous ulcers or bullae [9]. Finally, when there is a known trigger, it is important to counsel patients on avoidance. All 4 patients with alcohol-associated IgA vasculitis did not experience recurrence after abstaining from alcohol [3-5]. Non-steroidal anti-inflammatory drugs (NSAIDs) can be used in patients with mild to moderate joint or abdominal pain [16].

For severe pain and failed response to NSAIDs, systemic steroids have been used. Oral prednisone $1-2 \mathrm{mg} / \mathrm{kg} /$ day or equivalent doses of intravenous methylprednisolone is recommended with slow taper to prevent relapse of symptoms [17]. Systemic steroids have also been used in cases of scrotal swelling and arthritis [18]. For patients who relapse after systemic steroids, case studies on steroid-sparing agents (such as colchicine or dapsone) have demonstrated improvement during treatment and recurrence upon discontinuation [19-21]. Doses of 50-150 mg/day of dapsone are typically utilized with improvement often noted as early as 1 week after initiation of therapy. Potential adverse effects, including hemolytic anemia, must be carefully monitored in patients receiving dapsone [19, 20]. Colchicine for leukocytoclastic vasculitis is usually started as a $0.6-\mathrm{mg}$ dose twice daily, with initial response usually within 1-2 weeks [21]. Our patient was recommended avoidance initially given the resolution of extra-cutaneous symptoms prior to presentation. However, due to the patient's dislike of the appearance of the rash, he was prescribed topical triamcinolone followed by oral prednisone (particularly given the scrotal swelling and arthralgias present at subsequent flare) without significant improvement of the purpuric eruption. Those patients with significant renal disease, gastrointestinal bleeding, mobility-limiting joint pain, or changes in mental status, should be managed through a multidisciplinary approach.

In conclusion, alcohol-associated IgA vasculitis is a rare but important entity for dermatologists and dermatopathologists to be aware of in order to ensure appropriate management, counseling, and prevention of recurrent episodes of disease. To our knowledge, ours is the only case of alcohol-associated vasculitis triggered specifically by hops and the first case reported with extra-cutaneous symptoms and use of specific therapeutic agents.

\section{Statement of Ethics}

All subjects have given written informed consent to publish this case (including publication of images).

\section{Disclosure Statement}

The authors have no conflicts of interest to declare. 
Funding Sources

No funding was received.

\section{Author Contributions}

Study design: P.B. and S.D. Drafting of the manuscript: P.B. and S.D. Critical revision of the manuscript for important intellectual content: R.M.N. and E.R.-G. Administrative or technical support: S.D. Study supervision: S.D.

\section{References}

1 Saulsbury FT. Henoch-Schönlein purpura. Curr Opin Rheumatol. 2010 Sep;22(5):598-602.

2 Pillebout E, Thervet E, Hill G, Alberti C, Vanhille P, Nochy D. Henoch-Schönlein Purpura in adults: outcome and prognostic factors. J Am Soc Nephrol. 2002 May;13(5):1271-8.

3 Abuabara K, Samimi S, Chu EY, Bluebond ND, James WD, Merkel PA. Alcohol-induced vasculitis: case report and commentary. J Am Acad Dermatol. 2014 Feb;70(2):e42-3.

4 Chua IC, Aldridge CR, Finlay AY, Williams PE. Cutaneous IgA-associated vasculitis induced by alcohol. Br Dermatol. 2005 Nov;153(5):1037-40.

5 Alibrandi B, Parodi A, Varaldo G. Purpura due to ethanol. N Engl J Med. 1990 Mar;322(10):702.

6 Piram M, Mahr A. Epidemiology of immunoglobulin A vasculitis (Henoch-Schönlein): current state of knowledge. Curr Opin Rheumatol. 2013 Mar;25(2):171-8.

7 Johnson EF, Wetter DA, Lehman JS, Hand JL, Davis DM, Tollefson MM. Leukocytoclastic vasculitis in children: clinical characteristics, subtypes, causes and direct immunofluorescence findings of 56 biopsy-confirmed cases. J Eur Acad Dermatol Venereol. 2017 Mar;31(3):544-9.

8 Carlson JA, Ng BT, Chen KR. Cutaneous vasculitis update: diagnostic criteria, classification, epidemiology, etiology, pathogenesis, evaluation and prognosis. Am J Dermatopathol. 2005 Dec;27(6):504-28.

9 Fiorentino DF. Cutaneous vasculitis. J Am Acad Dermatol. 2003 Mar;48(3):311-40.

10 Russell JP, Gibson LE. Primary cutaneous small vessel vasculitis: approach to diagnosis and treatment. Int J Dermatol. 2006 Jan;45(1):3-13.

11 Jithpratuck W, Elshenawy Y, Saleh H, Youngberg G, Chi DS, Krishnaswamy G. The clinical implications of adultonset henoch-schonelin purpura. Clinical and Molecularl Allergy. 2011 May;9. https://doi.org/10.1186/14767961-9-9.

12 Coppo R, Andrulli S, Amore A, Gianoglio B, Conti G, Peruzzi L, et al. Predictors of outcome in Henoch-Schönlein nephritis in children and adults. Am J Kidney Dis. 2006 Jun;47(6):993-1003.

13 Larson AR, Granter SR. Utility of immunofluorescence testing for vascular IgA in adult patients with leukocytoclastic vasculitis. Am J Clin Pathol. 2014 Sep;142(3):370-4.

14 Demirkesen C. Approach to cutaneous vasculitides with special emphasis on small vessel vasculitis: histopathology and direct immunofluorescence. Curr Opin Rheumatol. 2017 Jan;29(1):39-44.

15 Deacock SJ. An approach to the patient with urticaria. Clin Exp Immunol. 2008 Aug;153(2):151-61.

16 Chen KR, Carlson JA. Clinical approach to cutaneous vasculitis. Am J Clin Dermatol. 2008;9(2):71-92.

17 Weiss PF, Feinstein JA, Luan X, Burnham JM, Feudtner C. Effects of corticosteroid on Henoch-Schönlein purpura: a systematic review. Pediatrics. 2007 Nov;120(5):1079-87.

18 Modi S, Mohan M, Jennings A. Acute Scrotal Swelling in Henoch-Schonlein Purpura: Case Report and Review of the Literature. Urol Case Rep. 2016 Feb;6:9-11.

19 Fredenberg MF, Malkinson FD. Sulfone therapy in the treatment of leukocytoclastic vasculitis. Report of three cases. J Am Acad Dermatol. 1987 Apr;16(4):772-8.

20 Fortson JS, Zone JJ, Hammond ME, Groggel GC. Hypocomplementemic urticarial vasculitis syndrome responsive to dapsone. J Am Acad Dermatol. 1986 Nov;15(5 Pt 2):1137-42.

21 Plotnick S, Huppert AS, Kantor G. Colchicine and leukocytoclastic vasculitis. Arthritis Rheum. 1989 Nov; 32(11):1489-90. 